\title{
THE LEBESGUE FUNCTION FOR GENERALIZED HERMITE-FEJÉR INTERPOLATION ON THE CHEBYSHEV NODES
}

\author{
GRAEME J. BYRNE ${ }^{1}$, T. M. MILLS ${ }^{1}$ and SIMON J. SMITH ${ }^{1}$
}

Dedicated to Prof. David Elliott on the occasion of his 65th birthday, in appreciation of his active encouragement during our mathematical careers

(Received 16 March 1998)

\begin{abstract}
This paper presents a short survey of convergence results and properties of the Lebesgue function $\lambda_{m, n}(x)$ for $(0,1, \ldots, m)$ Hermite-Fejér interpolation based on the zeros of the $n$th Chebyshev polynomial of the first kind. The limiting behaviour as $n \rightarrow \infty$ of the Lebesgue constant $\Lambda_{m, n}=\max \left\{\lambda_{m, n}(x):-1 \leq x \leq 1\right\}$ for even $m$ is then studied, and new results are obtained for the asymptotic expansion of $\Lambda_{m, n}$. Finally, graphical evidence is provided of an interesting and unexpected pattern in the distribution of the local maximum values of $\lambda_{m, n}(x)$ if $m \geq 2$ is even.
\end{abstract}

\section{Introduction}

Suppose $X=\left\{x_{k, n}: k=1,2, \ldots, n ; n=1,2,3, \ldots\right\}$ is a triangular array of nodes such that for each $n$

$$
1 \geq x_{1, n}>x_{2, n}>\cdots>x_{n, n} \geq-1,
$$

and let $f$ be a continuous real-valued function defined on the interval $[-1,1]$. Then, for each integer $m \geq 0$, there exists a unique polynomial $H_{m, n}(X, f, x)$ of degree at most $(m+1) n-1$ which satisfies

$$
H_{m, n}^{(r)}\left(X, f, x_{k, n}\right)=\delta_{0, r} f\left(x_{k, n}\right), \quad 1 \leq k \leq n, 0 \leq r \leq m .
$$

\footnotetext{
${ }^{1}$ Division of Mathematics, La Trobe University, P.O. Box 199, Bendigo, VIC 3552, Australia.

(C) Australian Mathematical Society 2000, Serial-fee code 0334-2700/00
} 
$H_{m, n}(X, f, x)$ is known as the $(0,1, \ldots, m)$ Hermite-Fejér (HF) interpolation polynomial of $f(x)$, and it can be expressed as

$$
H_{m, n}(X, f, x)=\sum_{k=1}^{n} f\left(x_{k, n}\right) A_{k, m, n}(X, x),
$$

where $A_{k, m, n}(X, x)$ is the unique polynomial of degree at most $(m+1) n-1$ such that

$$
A_{k, m, n}^{(r)}\left(X, x_{j, n}\right)=\delta_{0, r} \delta_{k, j}, \quad 1 \leq k, j \leq n, 0 \leq r \leq m .
$$

The $A_{k, m, n}(X, x)$ are referred to as the fundamental polynomials for $(0,1, \ldots, m) \mathrm{HF}$ interpolation on $X$. The function

$$
\lambda_{m, n}(X, x)=\sum_{k=1}^{n}\left|A_{k, m, n}(X, x)\right|
$$

and the quantity

$$
\Lambda_{m, n}(X)=\max _{-1 \leq x \leq 1} \lambda_{m, n}(X, x),
$$

which are known as the Lebesgue function and Lebesgue constant, respectively, for $(0,1, \ldots, m)$ HF interpolation on $X$, play a fundamental role in discussion of the convergence of $H_{m, n}(X, f, x)$ to $f(x)$ as $n \rightarrow \infty$.

Now, $H_{0, n}(X, f, x)$ is the well-known Lagrange interpolation polynomial of $f(x)$. For Lagrange interpolation it is known (see Rivlin [14, Section 1.3] and the references therein) that there exists a constant $c$, with $1 / 2<c<3 / 4$, such that

$$
\Lambda_{0, n}(X)>\frac{2}{\pi} \log n+c, \quad n=1,2,3, \ldots,
$$

for any $X$. By the principle of uniform boundedness, a consequence of (2) is the classic result, due to Faber [7], that for any matrix $X$, there exists $f \in C[-1,1]$ so that $H_{0, n}(X, f, x)$ does not tend uniformly to $f(x)$ on $[-1,1]$ as $n \rightarrow \infty$. This result, and other key developments in the history of the convergence theory for Lagrange interpolation, are described in the very readable paper by Elliott [6], while the monograph by Szabados and Vértesi [23] offers a more recent (and more technical) discussion of these matters.

Although Faber's result is quite negative in character, more positive results are available if particular node systems are chosen. For instance, if $T$ denotes the matrix of Chebyshev nodes

$$
T=\left\{\cos \left(\frac{2 k-1}{2 n} \pi\right): k=1,2, \ldots, n ; n=1,2,3, \ldots\right\},
$$


then

$$
\Lambda_{0, n}(T) \leq \frac{2}{\pi} \log n+1, \quad n=1,2,3, \ldots
$$

(See Rivlin [14, Theorem 1.2] for a proof of this result.) Thus the Chebyshev node system provides a simple set of nodes whose Lebesgue constants are close to best possible. Further, if the modulus of continuity $\omega(\delta ; f)$ of $f$ is defined by

$$
\omega(\delta ; f)=\max \{|f(s)-f(t)|:\{s, t\} \subset[-1,1],|s-t| \leq \delta\},
$$

then from (3) (cf. Rivlin [13, Section 4.1]) it follows that if $f \in C[-1,1]$ satisfies the relatively weak condition $\omega(1 / n ; f) \log n \rightarrow 0$ as $n \rightarrow \infty$, then the sequence of Lagrange interpolation polynomials $H_{0, n}(T, f, x)$ converges uniformly to $f(x)$ on $[-1,1]$ as $n \rightarrow \infty$. In view of these results, it can be seen that the Chebyshev nodes $T$ are a good choice if uniform approximation by Lagrange interpolation polynomials is required.

A key step in the proof of (3) is the observation, proved by Ehlich and Zeller [5], that

$$
\Lambda_{0, n}(T)=\lambda_{0, n}(T, \pm 1)
$$

A detailed analysis of the representation

$$
\lambda_{0, n}(T, 1)=\frac{1}{n} \sum_{k=1}^{n} \cot \frac{(2 k-1) \pi}{4 n}
$$

then leads to the asymptotic expansion (established independently by Dzjadyk and Ivanov [4], Günttner [9, 10] and Shivakumar and Wong [19])

$$
\Lambda_{0, n}(T)=\frac{2}{\pi} \log n+C_{0}+\frac{8}{\pi} \sum_{k=1}^{\infty} \frac{(-1)^{k+1} C_{k}}{(2 n)^{2 k}} .
$$

Here

$$
C_{k}= \begin{cases}\frac{2}{\pi}\left(\gamma+\log \frac{8}{\pi}\right), & k=0, \\ \left(2^{2 k-1}-1\right)^{2} \frac{\pi^{2 k}}{2 k} \frac{B_{2 k}^{2}}{(2 k) !}, & k \geq 1,\end{cases}
$$

where $\gamma$ denotes the Euler-Mascheroni constant, and the $B_{k}$ are the Bernoulli numbers. Further, the error in truncating the series on the right-hand side of (5) has the same sign as, and has absolute value less than, the first term neglected. For a more detailed survey 
of properties of the Lebesgue function and constant for Lagrange interpolation on $T$ (and on other node systems), the reader is referred to the recent paper by Brutman [2].

The initial motivation for generalising Lagrange interpolation to $(0,1, \ldots, m)$ HF interpolation $(m \geq 1)$ came from Fejér's result [8] that $H_{1, n}(T, f, x) \rightarrow f(x)$ uniformly in $[-1,1]$ for all $f \in C[-1,1]$. Thus the $(0,1)$ HF process has better uniform convergence properties than the Lagrange method, at least on the node system $T$. A key step in Fejér's proof was the observation that the fundamental polynomials $A_{k, 1, n}(T, x)$ are non-negative for $-1 \leq x \leq 1$. For the Lebesgue function, this has the consequence that

$$
\lambda_{1, n}(T, x)=\sum_{k=1}^{n} A_{k, 1, n}(T, x),
$$

where the right-hand side is a polynomial of degree $2 n-1$ or less which assumes the value 1 , and has vanishing derivative, at each of the $n$ Chebyshev nodes. By uniqueness considerations, $\lambda_{1, n}(T, x)$ is identically 1 , and so the issue of the Lebesgue constant for $(0,1) \mathrm{HF}$ interpolation on $T$ is resolved immediately.

For $(0,1,2) \mathrm{HF}$ interpolation, Szabados and Varma [22] showed there is a positive constant $c_{1}$ so that for any system of nodes $X$,

$$
\Lambda_{2, n}(X) \geq c_{1} \log n .
$$

This result was extended by Szabados [21], who showed there are constants $c_{m}>0$ so that

$$
\Lambda_{2 m, n}(X) \geq c_{m} \log n, \quad m=0,1,2, \ldots .
$$

Thus, for any system of nodes $X$, there exists $f \in C[-1,1]$ so that $H_{2 m, n}(X, f, x)$ does not converge uniformly to $f(x)$ on $[-1,1]$ as $n \rightarrow \infty$. Szabados also showed that the order of magnitude on the right-hand side of (6) is best possible, because there exist constants $d_{m}$ so that

$$
\Lambda_{2 m, n}(T) \leq d_{m} \log n, \quad m=0,1,2, \ldots .
$$

These results illustrate the generally observed fact that the Lagrange interpolation process and the Hermite-Fejér processes of even order share many properties in common.

An extensive study of $(0,1, \ldots, m) \mathrm{HF}$ interpolation on the Chebyshev nodes (and on their generalization, the Jacobi nodes) has appeared in papers by Sakai $[15,16]$, Vértesi $[24,25]$ and Sakai and Vértesi $[17,18]$. Among other results, they showed that if $m$ is odd, then $H_{m, n}(T, f, x) \rightarrow f(x)$ uniformly in $[-1,1]$ for all $f \in C[-1,1]$, and hence the Lebesgue constants $\Lambda_{m, n}(T)$ are uniformly bounded in $n$. This latter 
observation was refined recently by Smith [20], who established that if $m$ is odd, the fundamental polynomials $A_{k, m, n}(T, x)$ are non-negative for $-1 \leq x \leq 1$, and so the Lebesgue function $\lambda_{m, n}(T, x)$ is 1 for all $x$. Results such as these illustrate the principle that the Hermite-Fejér processes of odd order tend to have similar properties to those of the original Hermite-Fejér method (that is, to $(0,1) \mathrm{HF}$ interpolation).

If we return the discussion to that of the Hermite-Fejér processes of even order, Byrne, Mills and Smith [3] were able to generalize (4) by showing that for $m=$ $0,1,2, \ldots$,

$$
\Lambda_{2 m, n}(T)=\lambda_{2 m, n}(T, \pm 1)=\sum_{k=1}^{n}(-1)^{k-1} A_{k, 2 m, n}(T, 1) .
$$

By using estimates for the coefficients in the fundamental polynomials $A_{k, 2 m, n}(T, x)$ that were developed by Sakai and Vértesi $[17,18]$, they were also able to obtain the asymptotic result as $n \rightarrow \infty$,

$$
\Lambda_{2 m, n}(T)=\frac{2}{\pi} \frac{(2 m) !}{2^{2 m}(m !)^{2}} \log n+O(1) .
$$

Now, the coefficient estimates of Sakai and Vértesi that were used to develop (8) are in fact valid for $(0,1, \ldots, 2 m) \mathrm{HF}$ interpolation on a wide class of Jacobi nodes, and not just for interpolation on the Chebyshev nodes. This suggests that significant improvements to the asymptotic result (8) will follow only if a different approach to estimating the $A_{k, 2 m, n}(T, 1)$ is employed, whereby properties specific to the Chebyshev nodes are used. We have been able to achieve this by using explicit formulas for Hermite trigonometric interpolation based on equidistant points that were developed by KreB [11]. The following results, which are proved in Section 2 of this paper, have been obtained.

THEOREM 1. Suppose the constants $a_{r}=a_{r, m}$ are the coefficients in the Laurent expansion

$$
\frac{1}{\sin ^{2 m+1} \theta}=\frac{1}{\theta^{2 m+1}} \sum_{r=0}^{\infty} a_{r} \theta^{2 r}, \quad 0<|\theta|<\pi,
$$

and let

$$
\theta_{k}=\theta_{k, n}=\frac{2 k-1}{2 n} \pi, \quad k=1,2, \ldots, n ; n=1,2,3, \ldots .
$$

Then, for $m=0,1,2, \ldots$, the Lebesgue constant $\Lambda_{2 m, n}(T)$ can be written as

$$
\Lambda_{2 m, n}(T)=\sum_{k=1}^{n} \sum_{r=0}^{m} \frac{a_{m-r}}{n^{2 r+1}(2 r) !}\left[\frac{d^{2 r}}{d \theta^{2 r}} \cot \frac{\theta}{2}\right]_{\theta=\theta_{k}} .
$$


THEOREM 2. If $\zeta(k)$ denotes the Riemann zeta function $\zeta(k)=\sum_{n=1}^{\infty} n^{-k}$, then as $n \rightarrow \infty$,

$$
\begin{aligned}
\Lambda_{2 m, n}(T)= & \frac{2}{\pi} \frac{(2 m) !}{2^{2 m}(m !)^{2}} \log n+\frac{2}{\pi} \frac{(2 m) !}{2^{2 m}(m !)^{2}}\left(\gamma+\log \left(\frac{8}{\pi}\right)\right) \\
& +2 \sum_{r=1}^{m} a_{m-r} \frac{2^{2 r+1}-1}{\pi^{2 r+1}} \zeta(2 r+1)+O\left(n^{-2}\right) .
\end{aligned}
$$

\section{Proofs of Theorems 1 and 2}

PROOF OF THEOREM 1. Consider the cosine polynomial $t_{k, 2 m, n}(\theta)$, of degree no greater than $(2 m+1) n-1$, which is defined by

$$
t_{k, 2 m, n}(\theta)=A_{k, 2 m, n}(T, \cos \theta) .
$$

Then for $1 \leq k \leq n, t_{k, 2 m, n}(\theta)$ satisfies

$$
t_{k, 2 m, n}^{(r)}\left(\theta_{j}\right)=\delta_{0, r} \delta_{k, j}, \quad 1 \leq j \leq n, 0 \leq r \leq 2 m
$$

and is the unique cosine polynomial of degree $(2 m+1) n-1$ or less which has these properties. Further, from (7),

$$
\Lambda_{2 m, n}(T)=\sum_{k=1}^{n}(-1)^{k-1} t_{k, 2 m, n}(0)
$$

Now, by Kreß [11, Theorem 1.1], there is a unique trigonometric polynomial $S_{2 m, n}(\theta)$ of the form

$$
S_{2 m, n}(\theta)=\sum_{k=0}^{(2 m+1) n} p_{k} \cos k \theta+\sum_{k=1}^{(2 m+1) n-1} q_{k} \sin k \theta
$$

such that

$$
S_{2 m, n}^{(r)}(j \pi / n)=\delta_{0, r} \delta_{0, j}, \quad 0 \leq j \leq 2 n-1,0 \leq r \leq 2 m .
$$

Since $\left(S_{2 m, n}(\theta)+S_{2 m, n}(-\theta)\right) / 2$ also has these properties, it follows that $S_{2 m, n}(\theta)$ is even. Next, for $1 \leq k \leq n$ consider the function

$$
s_{k, 2 m, n}(\theta)=S_{2 m, n}\left(\theta-\theta_{k}\right)+S_{2 m, n}\left(\theta+\theta_{k}\right),
$$

which is even. Thus $s_{k, 2 m, n}(\theta)$ is a cosine polynomial of degree no greater than $(2 m+1) n$. However, direct calculation shows that the coefficient of $\cos (2 m+1) n \theta$ 
in $s_{k, 2 m, n}(\theta)$ is zero, and so $s_{k, 2 m, n}(\theta)$ is a cosine polynomial of degree $(2 m+1) n-1$ or less which satisfies

$$
s_{k, 2 m, n}^{(r)}\left(\theta_{j}\right)=\delta_{0, r} \delta_{k, j}, \quad 1 \leq j \leq n, 0 \leq r \leq 2 m .
$$

By the uniqueness properties of $t_{k, 2 m, n}(\theta)$ it follows that $t_{k, 2 m, n}(\theta)=s_{k, 2 m, n}(\theta)$, and so from (12) and (13) we obtain the representation

$$
\Lambda_{2 m, n}(T)=2 \sum_{k=1}^{n}(-1)^{k-1} S_{2 m, n}\left(\theta_{k}\right)
$$

An explicit formula for $S_{2 m, n}(\theta)$ is

$$
S_{2 m, n}(\theta)=\frac{\sin ^{2 m+1} n \theta}{2} \sum_{r=0}^{m} \frac{a_{m-r}}{n^{2 r+1}(2 r) !} \frac{d^{2 r}}{d \theta^{2 r}} \cot \frac{\theta}{2},
$$

where the $a_{m-r}$ are defined by (9) (see Kreß [11, Theorem 1.1]). From this formula and the representation (14), the required expression (10) for the Lebesgue constant $\Lambda_{2 m, n}(T)$ follows immediately.

PROOF OF THEOREM 2. We begin with the well-known expansion

$$
\cot \theta=\frac{1}{\theta}-\sum_{j=1}^{\infty} \frac{2^{2 j}\left|B_{2 j}\right|}{(2 j) !} \theta^{2 j-1}, \quad 0<|\theta|<\pi .
$$

Thus

$$
\frac{d^{2 r}}{d \theta^{2 r}} \cot \frac{\theta}{2}=\frac{2(2 r) !}{\theta^{2 r+1}}-\sum_{j=r+1}^{\infty} \frac{\left|B_{2 j}\right|}{j(2 j-2 r-1) !} \theta^{2 j-2 r-1}, \quad 0<|\theta|<2 \pi,
$$

and so by (10),

$$
\begin{aligned}
\Lambda_{2 m, n}(T)= & \sum_{k=1}^{n} \sum_{r=0}^{m} \frac{2 a_{m-r}}{(2 k-1)^{2 r+1}}\left(\frac{2}{\pi}\right)^{2 r+1} \\
& -\sum_{k=1}^{n} \sum_{r=0}^{m} \sum_{j=r+1}^{\infty} \frac{a_{m-r}\left|B_{2 j}\right|}{j(2 j-2 r-1) !(2 r) !} \frac{(2 k-1)^{2 j-2 r-1}}{n^{2 j}}\left(\frac{\pi}{2}\right)^{2 j-2 r-1} \\
= & S_{1}-S_{2}
\end{aligned}
$$

say, where $S_{1}$ denotes the double sum and $S_{2}$ the triple sum. To interpret the right-hand side of (16) we will employ the results ( $c f$. Günttner [9] or Shivakumar and Wong [19])

$$
\sum_{k=1}^{n} \frac{1}{2 k-1}=\frac{1}{2} \log n+\log 2+\frac{\gamma}{2}+\delta(n), \quad 0 \leq \delta(n) \leq \frac{1}{48 n^{2}},
$$




$$
\begin{gathered}
\sum_{k=1}^{n} \frac{1}{(2 k-1)^{2 r+1}}=\left(1-\frac{1}{2^{2 r+1}}\right) \zeta(2 r+1)-\phi_{r}(n), \\
0 \leq \phi_{r}(n) \leq \frac{1}{4 r(2 n)^{2 r}}, \quad r \geq 1,
\end{gathered}
$$

and, for $j \geq r+1$,

$$
\begin{aligned}
\sum_{k=1}^{n}(2 k-1)^{2 j-2 r-1} & =\frac{2^{2 j-2 r-2}}{j-r} n^{2 j-2 r}-\psi_{j, r}(n), \\
0 & \leq \psi_{j, r}(n) \leq \frac{2 j-2 r-1}{12}(2 n)^{2 j-2 r-2} .
\end{aligned}
$$

Now, from (17) and (18), it follows that the term $S_{1}$ on the right-hand side of (16) can be written in the form

$$
S_{\mathrm{l}}=\frac{2}{\pi} a_{m} \log n+\frac{2}{\pi} a_{m}(\gamma+\log 4)+2 \sum_{r=1}^{m} a_{m-r} \frac{2^{2 r+1}-1}{\pi^{2 r+1}} \zeta(2 r+1)+\varepsilon(n),
$$

where, by the estimates for $\delta(n)$ and $\phi(n)$ in (17) and (18), it is evident that

$$
\varepsilon(n)=2\left(\frac{2 a_{m}}{\pi} \delta(n)-\sum_{r=1}^{m}\left(\frac{2}{\pi}\right)^{2 r+1} a_{m-r} \phi_{r}(n)\right)=O\left(n^{-2}\right) .
$$

With regard to the triple sum $S_{2}$ on the right-hand side of (16), by (19) we can write

$$
\begin{aligned}
S_{2}= & \sum_{r=0}^{m} \frac{2 a_{m-r}}{\pi^{2 r+1}(2 r) !}\left(\sum_{j=r+1}^{\infty} \frac{\left|B_{2 j}\right| \pi^{2 j}}{2 j(2 j-2 r) !}\right) \frac{1}{n^{2 r}} \\
& -\sum_{r=0}^{m} \sum_{j=r+1}^{\infty} \frac{a_{m-r}\left|B_{2 j}\right|}{j(2 j-2 r-1) !(2 r) !}\left(\frac{\pi}{2}\right)^{2 j-2 r-1} \psi_{j, r}(n) \frac{1}{n^{2 j}} \\
= & S_{3}-S_{4},
\end{aligned}
$$

say. For $S_{3}$, we use the expansion (obtained by integrating (15))

$$
\log (\sin \theta)=\log \theta-\sum_{j=1}^{\infty} \frac{2^{2 j}\left|B_{2 j}\right|}{2 j(2 j) !} \theta^{2 j}, \quad 0<\theta<\pi
$$

to obtain

$$
S_{3}=\frac{2 a_{m}}{\pi} \log \left(\frac{\pi}{2}\right)+O\left(n^{-2}\right)
$$

For $S_{4}$, it follows from the estimate for $\psi_{j, r}(n)$ in (19) that

$$
0 \leq S_{4} \leq \frac{1}{12 \pi} \sum_{r=0}^{m} \frac{a_{m-r}}{\pi^{2 r}(2 r) !}\left(\sum_{j=r+1}^{\infty} \frac{\left|B_{2 j}\right| \pi^{2 j}}{2 j(2 j-2 r-2) !}\right) \frac{1}{n^{2 r+2}}=O\left(n^{-2}\right)
$$


Hence

$$
S_{2}=\frac{2 a_{m}}{\pi} \log \left(\frac{\pi}{2}\right)+O\left(n^{-2}\right) .
$$

If this result and the results (20) and (21) are substituted into (16), the required expansion (11) will be obtained, provided it can be shown that

$$
a_{m}=\frac{(2 m) !}{2^{2 m}(m !)^{2}} .
$$

One way of verifying (22) is to note that by (9), $a_{m}$ is the residue of $\sin ^{-(2 m+1)} \theta$ at 0 . Thus if $C_{R}$ denotes the rectangle with sides $x= \pm \pi / 2$ and $y= \pm i R$, and we let $R \rightarrow \infty$, a standard application of the residue theorem shows that

$$
a_{m}=\frac{1}{2 \pi i} \int_{C_{R}} \frac{1}{\sin ^{2 m+1} z} d z=\frac{2}{\pi} \int_{0}^{\infty} \frac{1}{\cosh ^{2 m+1} x} d x .
$$

This latter integral can be evaluated by substituting $u=(\cosh x)^{-2}$, and then the proof of Theorem 2 is complete.

REMARK. By using more precise asymptotic expansions than those in (17)-(19) (see Shivakumar and Wong [19]), the result (11) can be improved to an expansion of the form

$$
\begin{aligned}
\Lambda_{2 m, n}(T)= & \frac{2}{\pi} \frac{(2 m) !}{2^{2 m}(m !)^{2}} \log n+\frac{2}{\pi} \frac{(2 m) !}{2^{2 m}(m !)^{2}}\left(\gamma+\log \left(\frac{8}{\pi}\right)\right) \\
& +2 \sum_{r=1}^{m} a_{m-r} \frac{2^{2 r+1}-1}{\pi^{2 r+1}} \zeta(2 r+1)+\sum_{\ell=1}^{s-1} \frac{c_{\ell, m}}{n^{2 \ell}}+O\left(n^{-2 s}\right) .
\end{aligned}
$$

A result of this form for $\Lambda_{2, n}(T)$ is given by Byrne, Mills and Smith [3, Theorem 3].

\section{Local maxima of the Lebesgue function}

For any system of nodes $X=\left\{x_{k, n}: k=1,2, \ldots, n ; n=1,2,3, \ldots\right\}$ satisfying (1), it is known (see, for example, Luttmann and Rivlin [12]) that for $n \geq 3$ the Lebesgue function $\lambda_{0, n}(X, x)$ for Lagrange interpolation is a piecewise polynomial satisfying $\lambda_{0, n}(X, x) \geq 1$, with equality only at the nodes $x_{k, n}(k=1,2, \ldots, n)$. Between consecutive nodes $\lambda_{0, n}(X, x)$ has a single maximum, while in $\left(-1, x_{n, n}\right)$ and $\left(x_{1, n}, 1\right)$, it is monotonic decreasing and increasing, respectively. These properies are illustrated in Figure 1, which shows a graph of the Lebesgue function $\lambda_{0,8}(T, x)$ for Lagrange interpolation on eight Chebyshev nodes. (The graph also illustrates the 


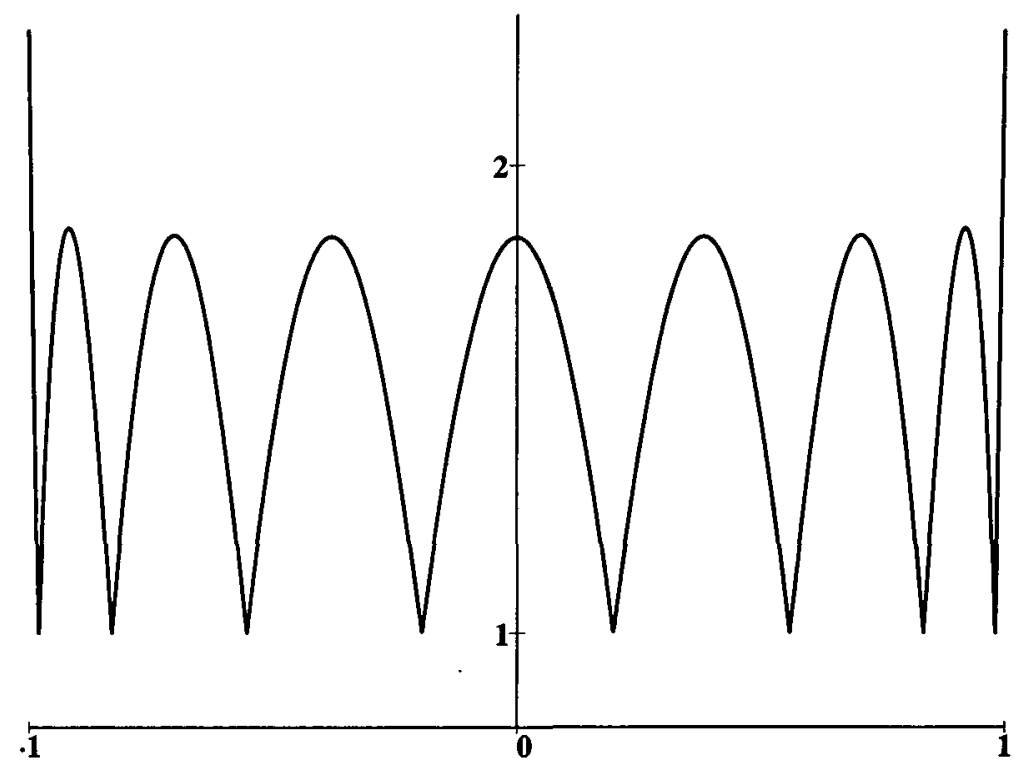

FIGURE 1. The Lebesgue function $\lambda_{0,8}(T, x)$

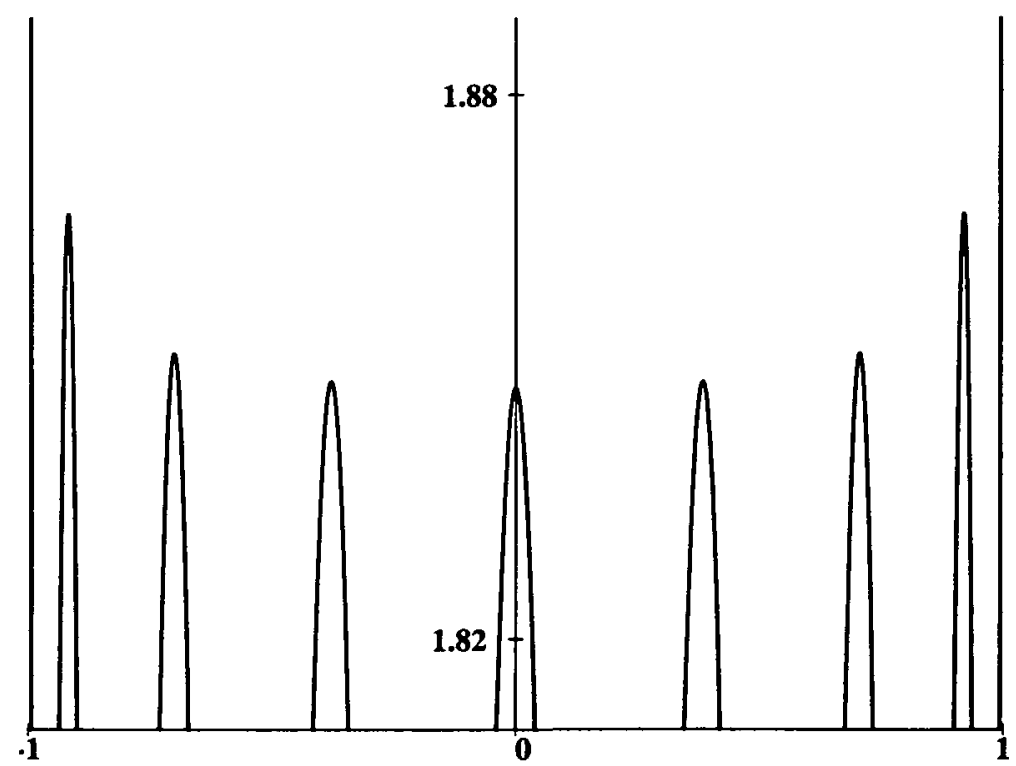

FIGURE 2. Section of the graph of $\lambda_{0,8}(T, x)$ 


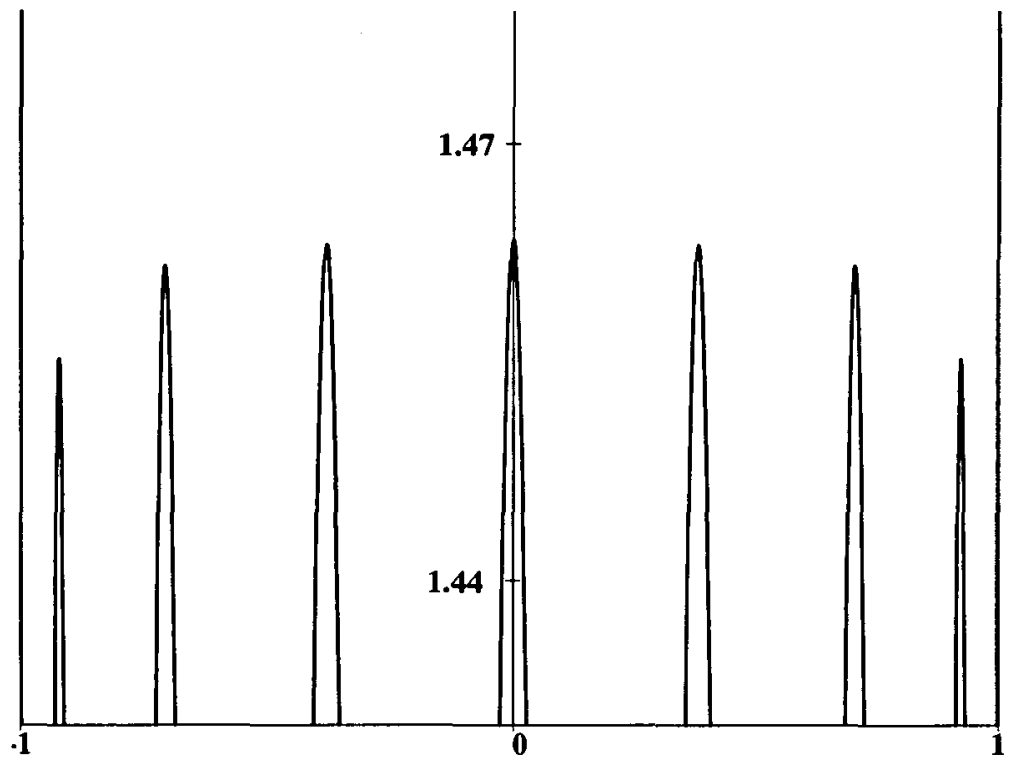

FIGURE 3. Section of the graph of $\lambda_{2,8}(T, x)$

result (4) that the maximum of $\lambda_{0, n}(T, x)$ on $[-1,1]$ occurs at \pm 1 . However, it is not true in general that the maximum of $\lambda_{0, n}(X, x)$ is achieved at \pm 1 .)

Figure 2 contains an enlargement of a section of the graph in Figure 1. It reveals an interesting pattern in the local maximum values of $\lambda_{0,8}(T, x)$, which appear to strictly decrease as we move from the endpoints towards the centre of the interval $[-1,1]$. This behaviour of the local maximum values of $\lambda_{0, n}(T, x)$ was observed (as a result of numerical computations) by Luttmann and Rivlin [12] and proved by Brutman [1]. (See also Günttner [9].)

Now, as noted earlier, the Lagrange interpolation method and the Hermite-Fejér processes of even order have many properties in common. However, this similarity does not seem to extend to the behaviour of the local maximum values of the Lebesgue function on the Chebyshev nodes. As an illustration of this, consider Figure 3, which contains a section of the graph of $\lambda_{2,8}(T, x)$. (The figure, like the earlier figures, was produced using the computer algebra system Maple.) The graph shows that the local maximum values in the interval $(-1,1)$ appear to increase as we move from the endpoints towards the centre of the interval. This is in direct contrast to the behaviour of the local maxima of $\lambda_{0, n}(T, x)$. It is interesting to conjecture whether this observed behaviour of the local maximum values of $\lambda_{2,8}(T, x)$ extends to $\lambda_{2 m, n}(T, x)$ for general $m \geq 1$ and $n \geq 3$. (Computer-generated graphs of $\lambda_{2 m, n}(T, x)$ for several values of $m$ and $n$ appear to support the conjecture.) 


\section{References}

[1] L. Brutman, "On the Lebesgue function for polynomial interpolation", SIAM J. Numer. Anal. 15 (1978) 694-704.

[2] L. Brutman, "Lebesgue functions for polynomial interpolation-a survey", Ann. Numer. Math. 4 (1997) 111-127.

[3] G. J. Byrne, T. M. Mills and S. J. Smith, "The Lebesgue constant for higher order Hermite-Fejér interpolation on the Chebyshev nodes", J. Approx. Theory 81 (1995) 347-367.

[4] V. K. Dzjadyk and V. V. Ivanov, "On asymptotics and estimates for the uniform norms of the Lagrange interpolation polynomials corresponding to the Chebyshev nodal points", Anal. Math. 9 (1983) 85-97.

[5] H. Ehlich and K. Zeller, "Auswertung der Normen von Interpolationsoperatoren", Math. Ann. 164 (1966) 105-112.

[6] D. Elliott, "Lagrange interpolation-decline and fall?", Int. J. Math. Educ. Sci. Technol. 10 (1979) $1-12$.

[7] G. Faber, "Über die interpolatorische Darstellung stetiger Funktionen", Jahresber. Deutsch. Math.Verein. 23 (1914) 190-210.

[8] L. Fejér, "Über Interpolation”, Göttinger Nachrichten (1916) 66-91.

[9] R. Günttner, "Evaluation of Lebesgue constants", SIAM J. Numer. Anal. 17 (1980) 512-520.

[10] R. Günttner, "On asymptotics for the uniform norms of the Lagrange interpolation polynomials corresponding to extended Chebyshev nodes", SIAM J. Numer. Anal. 25 (1988) 461-469.

[11] R. Kreß, "On general Hermite trigonometric interpolation", Numer. Math. 20 (1972) 125-138.

[12] F. W. Luttmann and T. J. Rivlin, "Some numerical experiments in the theory of polynomial interpolation", IBM J. Res. Develop. 9 (1965) 187-191.

[13] T. J. Rivlin, An introduction to the approximation of functions (Dover, New York, 1981).

[14] T. J. Rivlin, Chebyshev polynomials, 2nd ed. (Wiley, New York, 1990).

[15] R. Sakai, "Hermite-Fejér interpolation prescribing higher order derivatives", in: Progress in Approximation Theory (eds P. Nevai and A. Pinkus), (Academic Press, Boston, 1991), pp. 731759.

[16] R. Sakai, "Certain unbounded Hermite-Fejér interpolatory polynomial operators", Acta Math. Hungar. 59 (1992) 111-114.

[17] R. Sakai and P. Vértesi, "Hermite-Fejér interpolations of higher order. III", Studia Sci. Math. Hungar. 28 (1993) 87-97.

[18] R. Sakai and P. Vértesi, "Hermite-Fejér interpolations of higher order. IV", Studia Sci. Math. Hungar. 28 (1993) 379-386.

[19] P. N. Shivakumar and R. Wong, "Asymptotic expansion of the Lebesgue constants associated with polynomial interpolation", Math. Comp. 39 (1982) 195-200.

[20] S. J. Smith, "On the positivity of the fundamental polynomials for generalized Hermite-Fejer interpolation on the Chebyshev Nodes", J. Approx. Theory 96 (1999) 338-344.

[21] J. Szabados, "On the order of magnitude of fundamental polynomials of Hermite interpolation", Acta Math. Hungar. 61 (1993) 357-368.

[22] J. Szabados and A. K. Varma, "On $(0,1,2)$ interpolation in uniform metric", Proc. Amer. Math. Soc. 109 (1990) 975-979.

[23] J. Szabados and P. Vértesi, Interpolation of Functions (World Scientific, Singapore, 1990).

[24] P. Vértesi, "Hermite-Fejér interpolations of higher order. I", Acta Math. Hungar. 54 (1989) 135152.

[25] P. Vértesi, "Hermite and Hermite-Fejér interpolations of higher order. II (Mean convergence)", Acta Math. Hungar. 56 (1990) 369-379. 